\title{
Sleep EEG Characteristics in Young and Elderly Patients with Obstructive Sleep Apnea Syndrome
}

\author{
Yu Jin Lee ${ }^{1}$, Jong Won Kim²,3, Yu-Jin G. Lee ${ }^{4}$, and Do-Un Jeong ${ }^{1 凶}$ \\ ${ }^{1}$ Department of Psychiatry and Center for Sleep and Chronobiology, Seoul National University College of Medicine, Seoul, Republic of Korea \\ ${ }^{2}$ NHMRC Centre for Integrated Research and Understanding of Sleep, Woolcock Institute of Medical Research, The University of Sydney, \\ Sydney, Australia \\ ${ }^{3}$ School of Physics, The University of Sydney, Sydney, Australia \\ ${ }^{4}$ Department of Psychiatry, Seoul Metropolitan Eunpyeong Hospital, Seoul, Republic of Korea
}

\begin{abstract}
Objective In the present study, it was hypothesized that the sleep electroencephalogram (EEG) characteristics of young $(<30$ yrs $)$ and elderly ( $>55$ yrs) OSAS patients would differ.

Methods We analyzed 76 sleep EEG recordings from OSAS patients (young group: $n=40$, mean age: $24.3 \pm 4.9$ yrs; elderly group: $n=36$, mean age: $59.1 \pm 4.9 \mathrm{yrs}$ ), which were obtained during nocturnal polysomnography. The recordings were assessed via spectral analysis in the delta $(0.5-4.5 \mathrm{~Hz})$, theta $(4.5-8 \mathrm{~Hz})$, alpha $(8-12 \mathrm{~Hz})$, beta $(12-32 \mathrm{~Hz})$, slow sigma $(11-13 \mathrm{~Hz})$, and fast sigma $(13-17 \mathrm{~Hz})$ frequency bands.

Results Apnea Hypopnea Index (AHI) and sleep efficiency (\%) did not differ significantly between the two groups (19.8 \pm 14.4 vs. $25.9 \pm 16.0, p=0.085 ; 84.4 \pm 12.6$ vs. $80.9 \pm 11.0, p=0.198$, respectively). After adjusting for gender, the slow/fast sigma ratio was not significantly correlated with AHI in the elderly group $(\mathrm{r}=-0.047, \mathrm{p}=0.790)$ but AHI was inversely correlated with the slow/fast sigma ratio in the young group $(\mathrm{r}=-0.423, \mathrm{p}=0.007)$. A multiple linear regression analysis revealed that a higher AHI was related with a lower slow/fast sigma ratio in the young group $(\beta=-0.392, \mathrm{p}=0.028)$ but not the elderly.

Conclusion In the present study, sleep EEG activity differed between young and elderly OSAS patients. The slow/fast sigma ratio was associated with OSAS severity only in young patients, suggesting that young OSAS patients may have a distinctive brain plasticity compared with elderly patients.

Psychiatry Investig 2016;13(2):217-221
\end{abstract}

Key Words OSAS, Sleep EEG, Sleep spindle.

\section{INTRODUCTION}

Sleep fragmentation and oxygen desaturation in obstructive sleep apnea syndrome (OSAS) are known to reduce sleep quality and induce brain dysfunction. ${ }^{1,2}$ Sleep spindles, a wellknown microstructure of non-rapid eye movement (NREM) sleep, are considered to be markers of the thalamo cortical network ${ }^{3}$ and have been suggested as neurophysiological markers of sleep homeostasis. ${ }^{4}$ Sleep spindles are associated with the inhibition of sensory processing and gating of dis-

Received: May 8, 2015 Revised: June 13, 2015

Accepted: June 13, 2015 Available online: October 29, 2015

$\triangle$ Correspondence: Do-Un Jeong, MD, PhD

Department of Psychiatry and Center for Sleep and Chronobiology, Seoul National University College of Medicine, 103 Daehak-ro, Jongno-gu, Seoul 03080, Republic of Korea

Tel: +82-2-2072-2294, Fax: +82-2-744-7241, E-mail: jeongdu@snu.ac.kr

(c) This is an Open Access article distributed under the terms of the Creative Commons Attribution Non-Commercial License (http://creativecommons.org/licenses/bync/3.0) which permits unrestricted non-commercial use, distribution, and reproduction in any medium, provided the original work is properly cited. turbing intrusive stimuli and thereby maintain NREM sleep continuity. ${ }^{5}$ Additionally, sleep spindles play an important role in sleep-related brain functions such as memory consolidation and learning processes. ${ }^{3,6,7}$ and are regarded as sensitive electrophysiological markers of brain dysfunction in OSAS patients. $^{8}$

In previous studies, sleep spindles have been divided into two categories: fast spindles $(\geq 13 \mathrm{~Hz})$ and slow spindles $(<13$ $\mathrm{Hz}$ ). Slow and fast spindles undergo differing modulatory changes in the course of sleep ${ }^{9}$ and appear to have distinct functional properties. ${ }^{10}$ Although both types of spindles are involved in learning, fast spindles in particular activate the cerebral network involved in sleep-dependent memory consolidation. ${ }^{6}$

Recent findings indicate that changes in sleep spindles may be associated with various brain dysfunctions such as age-related changes, psychiatric illnesses, and OSAS. For example, spindle oscillatory frequency increases with age ${ }^{11}$ but decreases in OSAS. ${ }^{8,12}$ Similarly, Carvalho et al. ${ }^{13}$ reported the loss of 
physiological deceleration of sleep spindles in patients with OSAS.

OSAS is a common sleep disorder but tends to be more prevalent among the elderly population. ${ }^{14}$ Elderly individuals are more likely to have decreased sleep quality and may be more vulnerable to changes in sleep architecture, heart rate variability, ${ }^{15}$ and cognitive decline due to OSAS. ${ }^{16}$ Therefore, an investigation of differences in the electroencephalogram (EEG) characteristics of young and elderly people with OSAS, such as differences in sleep spindles, may provide important information regarding the changes in brain plasticity associated with aging. To our knowledge, no study has compared sleep EEG activity between young and elderly patients with OSAS. Thus, the present study hypothesized that sleep EEG characteristics, especially fast and slow sigma activities as indices of sleep spindle activation ${ }^{17}$ would differ between young and elderly OSAS patients.

\section{METHODS}

The present study reviewed the medical records of $347 \mathrm{pa}-$ tients aged under 30 years (young group) or over 55 years (elderly group) that had been diagnosed with OSAS using nocturnal polysomnography (Profusion PSG3, Compumedics) at the Sleep and Chronobiology Center of Seoul National University Hospital. A standard EEG montage and referential derivation were used for the nocturnal polysomnography procedure, which was initially scored by trained sleep technologists and then by sleep physicians, according to standard criteria. ${ }^{18}$ Apnea was defined as a complete or near-complete ( $\geq 90 \%$ ) cessation of airflow lasting $\geq 10$ s. Hypopnea was defined as a reduction in the respiratory signal (>30\%) lasting $\geq 10$ s that was associated with oxygen desaturation ( $\geq 4 \%)$. The Apnea Hypopnea Index (AHI) score was calculated as the average number of apnea and hypopnea events per hour of sleep and patients with scores higher than 5 were diagnosed with OSAS. Patients with any other medical illnesses such as diabetes, hypertension, or neurological disorders were exclud- ed from the present study, as were patients taking any medications or undergoing continuous positive airway pressure treatment. The nocturnal polysomnographic data for current analyses were from the baseline sleep studies for diagnostic processes. This study was approved by the Institutional Review Board at the Seoul National University Hospital.

Ultimately, 76 OSAS patients (young group: $n=40$, mean age: $24.3 \pm 4.9,87.5 \%$ male; elderly group: $n=36$, mean age: $59.1 \pm 4.9,77.8 \%$ male) were included in the final analyses. A central EEG channel at C3-A2 was primarily used for the quantitative EEG (qEEG) analyses; if C3-A2 was unavailable, or the recording from this location was of poor quality, then C4-A1 was used. A spectral analysis was performed for each 5-s epoch after removing non-neurogenic EEG artifacts using the qEEG-PSA program (CIRUS, Australia). ${ }^{19}$ Spectral power was obtained in the delta $(0.5-4.5 \mathrm{~Hz})$, theta $(4.5-8$ $\mathrm{Hz})$, alpha $(8-12 \mathrm{~Hz})$, beta $(12-32 \mathrm{~Hz})$, slow sigma (11-13 $\mathrm{Hz})$, and fast sigma $(13-17 \mathrm{~Hz})$ frequency bands and then averaged over each sleep stage and labeled accordingly (e.g., $\mathrm{N} 1, \mathrm{~N} 2, \mathrm{~N} 3$, etc.). Because the focus of the present study was sigma power, the mean spectral power in N2 and N3 for each band was calculated and used for the final analyses.

\section{Statistical analysis}

For between-group comparisons, independent t-tests were used for continuous variables and Fisher's exact tests were used for categorical variables. A partial correlation analysis controlling for gender was performed to assess the relationship between EEG power and AHI. Analyses were performed using SPSS 18.0 for Windows. All analyses were two-tailed, and a $\mathrm{p}$ value $<0.05$ was considered to indicate statistical significance.

\section{RESULTS}

No significant differences appeared in OSAS severity (AHI) or sleep efficiency $(\%)$ between the young and old groups $(\mathrm{p}=$ 0.085 and $\mathrm{p}=0.198$, respectively); Table 1 compares polysom-

Table 1. Comparison of polysomnographic variables between young and elderly OSAS patients

\begin{tabular}{lcccc}
\hline & Young group $(\mathrm{N}=40)$ & Elderly group $(\mathrm{N}=36)$ & t or $\chi^{2}$ & $\mathrm{p}$ \\
\hline Age & $24.3 \pm 4.9$ & $59.1 \pm 4.9$ & -30.698 & $<0.001^{* * *}$ \\
Gender (male \%) ${ }^{\dagger}$ & $35 / 5$ (male: $87.5 \%)$ & $28 / 8($ male: $77.8 \%)$ & 1.263 & 0.363 \\
Apnea-Hypopnea Index (AHI) & $19.8 \pm 14.4$ & $25.9 \pm 16.0$ & -1.744 & 0.085 \\
Sleep efficiency (\%) & $84.4 \pm 12.6$ & $80.1 \pm 11.0$ & 1.299 & 0.198 \\
N1 (\%) & $17.4 \pm 9.8$ & $23.6 \pm 10.7$ & 2.628 & $0.010^{*}$ \\
N2 (\%) & $47.0 \pm 9.7$ & $46.5 \pm 8.2$ & -0.238 & 0.813 \\
N3 (\%) & $14.9 \pm 9.2$ & $8.5 \pm 6.1$ & -3.482 & $0.001^{* *}$ \\
REM (\%) & $20.7 \pm 5.1$ & $21.4 \pm 5.3$ & 0.537 & 0.593 \\
\hline
\end{tabular}

Independent t-test. ${ }^{*} \mathrm{p}<0.05,{ }^{* *} \mathrm{p}<0.01,{ }^{* * *} \mathrm{p}<0.001,{ }^{\dagger}$ chi-squared test. AHI: Apnea-Hypopnea Index, OSAS: obstructive sleep apnea syndrome 
nographic variables between the two groups.

The young group exhibited significantly higher delta band activity $(\%)$ than the elderly group $(\mathrm{p}<0.001)$ but the elderly group exhibited more activity (\%) in the theta, alpha, and slow and fast sigma bands $(\mathrm{p}=0.002, \mathrm{p}<0.001, \mathrm{p}<0.001$, and $\mathrm{p}=$ 0.014 , respectively). No significant differences appeared between the groups in terms of the slow/fast sigma ratio $(\mathrm{p}=$ 0.648) (Table 2).

A partial correlation analysis adjusted for gender revealed that sleep efficiency was inversely correlated with $\mathrm{AHI}$ in the young group ( $\mathrm{p}=0.018$ ). Activities in the delta, theta, alpha, beta, and slow and fast sigma bands were not significantly correlated with $\mathrm{AHI}$ in the young group $(\mathrm{p}=0.206, \mathrm{p}=0.263, \mathrm{p}=$ $0.399, \mathrm{p}=0.337, \mathrm{p}=0.766$, and $\mathrm{p}=0.178$, respectively). The ratio of slow to fast sigma activity in the young group was inversely correlated with AHI ( $\mathrm{p}=0.007)$ (Table 3$)$.

In the partial correlation analysis adjusted for gender, sleep efficiency was not significantly correlated with AHI in the elderly group ( $p=0.552)$. Activities in the delta, theta, alpha, beta, and slow and fast sigma bands were not significantly correlated with $\mathrm{AHI}$ in the elderly group $(\mathrm{p}=0.876, \mathrm{p}=0.723$, $\mathrm{p}=$ $0.933, \mathrm{p}=0.987, \mathrm{p}=0.698$, and $\mathrm{p}=0.810$, respectively). Additionally, the ratio of slow to fast sigma activity was not significantly correlated with AHI in the elderly group ( $\mathrm{p}=0.790$ ) (Table 3 ).

In the multiple regression models adjusted for age, gender, and sleep efficiency, a higher AHI score was related with a lower slow/fast sigma ratio for the total subjects and the young group ( $\mathrm{p}=0.033$ and $\mathrm{p}=0.028$, respectively) but not the elderly group $(\mathrm{p}=0.841)($ Table 4$)$.

\section{DISCUSSION}

The present study demonstrated differences in sleep EEG activity between young and elderly patients with OSAS. In

Table 2. Comparison of EEG spectral activities between young and elderly OSAS patients

\begin{tabular}{lcccc}
\hline & Young group $(\mathrm{N}=40)$ & Elderly group $(\mathrm{N}=36)$ & $\mathrm{t}$ & $\mathrm{p}$ \\
\hline Delta activity (\%) & $88.6 \pm 5.5$ & $82.9 \pm 5.0$ & 4.667 & $<0.001^{* * *}$ \\
Theta activity (\%) & $5.9 \pm 3.8$ & $8.3 \pm 2.6$ & -3.165 & $0.002^{* *}$ \\
Alpha activity (\%) & $2.7 \pm 1.3$ & $5.0 \pm 1.7$ & -6.610 & $<0.001^{* * *}$ \\
Beta activity (\%) & $2.9 \pm 1.8$ & $3.9 \pm 2.5$ & -1.983 & 0.051 \\
Slow sigma activity (\%) & $1.1 \pm 0.6$ & $1.6 \pm 0.4$ & -3.775 & $<0.001^{* * *}$ \\
Fast sigma activity (\%) & $1.2 \pm 0.8$ & $1.7 \pm 0.6$ & -2.522 & $0.014^{*}$ \\
Slow/fast sigma ratio & $1.0 \pm 0.3$ & $1.0 \pm 0.2$ & -0.458 & 0.648 \\
\hline
\end{tabular}

Independent t-test. ${ }^{*} \mathrm{p}<0.05,{ }^{* *} \mathrm{p}<0.01,{ }^{* * *} \mathrm{p}<0.001$. EEG: electroencephalogram, OSAS: obstructive sleep apnea syndrome

Table 3. Correlations of AHI with sleep efficiency and EEG activities in young and elderly OSAS patients

\begin{tabular}{|c|c|c|c|c|}
\hline & & AHI in total group $(\mathrm{N}=76)$ & $\mathrm{AHI}$ in young group $(\mathrm{N}=40)$ & AHI in elderly group $(\mathrm{N}=36)$ \\
\hline \multirow[t]{2}{*}{ Sleep efficiency (\%) } & $\mathrm{r}$ & -0.273 & -0.378 & -0.104 \\
\hline & $\mathrm{p}$ & $0.018^{*}$ & $0.018^{*}$ & 0.552 \\
\hline \multirow[t]{2}{*}{ Delta activity (\%) } & $\mathrm{r}$ & -0.220 & -0.207 & -0.027 \\
\hline & $\mathrm{p}$ & 0.058 & 0.206 & 0.876 \\
\hline \multirow[t]{2}{*}{ Theta activity (\%) } & $\mathrm{r}$ & 0.207 & 0.184 & 0.062 \\
\hline & $\mathrm{p}$ & 0.075 & 0.263 & 0.723 \\
\hline \multirow[t]{2}{*}{ Alpha activity (\%) } & $\mathrm{r}$ & 0.180 & 0.139 & -0.015 \\
\hline & $\mathrm{p}$ & 0.121 & 0.399 & 0.933 \\
\hline \multirow[t]{2}{*}{ Beta activity (\%) } & $\mathrm{r}$ & 0.108 & 0.158 & -0.003 \\
\hline & $\mathrm{p}$ & 0.358 & 0.337 & 0.987 \\
\hline \multirow[t]{2}{*}{ Slow sigma activity (\%) } & $\mathrm{r}$ & 0.091 & -0.049 & 0.068 \\
\hline & $\mathrm{p}$ & 0.437 & 0.766 & 0.698 \\
\hline \multirow[t]{2}{*}{ Fast sigma activity (\%) } & $\mathrm{r}$ & 0.199 & 0.220 & 0.042 \\
\hline & $\mathrm{p}$ & 0.087 & 0.178 & 0.810 \\
\hline \multirow[t]{2}{*}{ Slow/fast sigma ratio } & $\mathrm{r}$ & -0.257 & -0.423 & -0.047 \\
\hline & $\mathrm{p}$ & $0.026^{*}$ & $0.007^{* *}$ & 0.790 \\
\hline
\end{tabular}

Partial correlation analysis was performed after adjusting for gender. ${ }^{*} \mathrm{p}<0.05,{ }^{* *} \mathrm{p}<0.01$. AHI: Apnea-Hypopnea Index, OSAS: obstructive sleep apnea syndrome, EEG: electroencephalogram 
Table 4. Multiple linear regression analysis predicting slow/fast sigma ratios in young and elderly OSAS

\begin{tabular}{cccc}
\hline $\begin{array}{c}\text { Independent } \\
\text { variables }\end{array}$ & $\begin{array}{c}\text { Total } \\
(\mathrm{N}=76)\end{array}$ & $\begin{array}{c}\text { Young group } \\
(\mathrm{N}=40)\end{array}$ & $\begin{array}{c}\text { Elderly group } \\
(\mathrm{N}=36)\end{array}$ \\
\hline Age & & & \\
$\beta$ & 0.124 & -0.089 & 0.271 \\
$\mathrm{p}$ & 0.298 & 0.571 & 0.155 \\
Gender & & & \\
$\beta$ & -0.008 & -0.062 & 0.137 \\
$\mathrm{p}$ & 0.947 & 0.694 & 0.471 \\
Sleep efficiency & & & \\
$\beta$ & 0.091 & 0.085 & 0.113 \\
$\mathrm{p}$ & 0.444 & 0.615 & 0.550 \\
AHI & & & -0.038 \\
$\beta$ & -0.272 & -0.392 & 0.841 \\
\hline $\mathrm{p}$ & $0.033^{*}$ & $0.028^{*}$ & \\
\hline
\end{tabular}

Dependent variable: slow/fast sigma ratio, Independent variables: age, gender, sleep efficiency, and AHI. ${ }^{*} \mathrm{p}<0.05$. AHI: Apnea-Hypopnea Index, OSAS: obstructive sleep apnea syndrome

particular, a significant association was observed between OSAS severity and the slow/fast sigma ratio, but only in the young group.

As expected, more delta activity was observed in the EEG recordings of the young OSAS patients compared with the elderly patients, whereas the elderly OSAS group exhibited more theta and alpha activities. These results are consistent with previous findings showing decreased slow waves and increased light sleep in elderly individuals. ${ }^{20}$ In the present study, more sigma activity was observed in the elderly patients compared with the young patients according to measurements of relative EEG power. The increased sigma activity in elderly patients may be related to a relative decrease in delta activity.

Sleep efficiency was inversely related to OSAS severity in the young group, which is partially consistent with the findings of a previous report that investigated low sleep efficiency in severe OSAS using nocturnal polysomnography. ${ }^{21}$ Respiratory events during sleep can induce sleep fragmentation due to the respiratory efforts that are associated with upper airway obstruction in OSAS patients, which decline with age. ${ }^{22}$ The absence of a correlation in the elderly group in the present study may indicate decreased respiratory effort, perhaps due to disruptions of neuromuscular compensation to hypoxemia caused by respiratory cessation in elderly patients.

Additionally, the slow/fast sigma ratio was related with OSAS severity in the young group. Sleep spindles are thought to be related to synaptic changes and long-term potentiation. ${ }^{3}$ Slow spindles $(<13 \mathrm{~Hz})$ predominate in frontal cortical areas, originate from the medial frontal region, and are associated with an increased activation in the superior frontal gyrus. In contrast, fast spindles $(\geq 13 \mathrm{~Hz})$ are dominant over the central and parietal areas, originate from the precuneus areas, and are associated with the activation of the hippocampus, medial frontal cortex, and brain areas involved in sensory-motor processing. ${ }^{23}$ Although some studies have demonstrated an association between slow spindles and cognitive performance, other studies have stressed the role of fast spindles in the consolidation of visuomotor ${ }^{24}$ and motor sequence learning processes. ${ }^{6}$ In terms of cognitive decline due to OSAS, the association of AHI with the slow/fast sigma ratio in young patients in the present study may suggest that there can be an activation of compensatory mechanisms based in brain plasticity. Thus, the decreased synaptic plasticity in the elderly patients due to the aging process may be reflected by the absence of an association between sigma activity and OSAS severity. Decreased brain compensation in the elderly patients may result in agerelated alterations of the thalamo-cortical regulatory mechanisms that are associated with sleep spindle production. ${ }^{11}$

It is also possible that the impaired synaptic plasticity in elderly patients in the present study may have been caused by their relatively longer duration of OSAS compared to young patients. OSAS may induce cerebral abnormalities, such as vascular dysfunctions, that can affect brain plasticity. ${ }^{2}$ Due to the limitations inherent in the retrospective design of the present study, the duration of OSAS was not controlled for. However, the duration of OSAS would not significantly influence the present results because all of the data analyzed in this study were baseline assessments intended for diagnosis. However, the effects of disease duration on neural plasticity should be a topic of interest in follow-up studies.

Ayalon et al..$^{25}$ noted that middle-aged patients with OSAS exhibit reduced performance in attention tasks compared with younger patients, suggesting that the concomitant presence of sleep apnea and increasing age overwhelms the brain's capacity to respond to cognitive tasks with compensatory recruitment. Therefore, the present results support the notion that elderly individuals may be more vulnerable to neurocognitive changes due to OSAS because of their reduced compensatory capacity that may be partially reflected by the absence of a significant correlation between OSAS severity and sigma activity. Additionally, the present results partially support the theory that sleep spindles may be a neurophysiological marker of brain synaptic plasticity in OSAS patients.

The present study has several limitations. First, a relatively small number of patients were assessed. Second, it used a sigma band that was broader than the range of classical sleep spindles; however, an accurate definition of the fast/slow spindle (sigma) band has yet to be well defined. Third, it did not include a control group for the purpose of comparison.

Despite these limitations, the present study had several 
strengths. None of the participants had any medical or psychiatric illnesses, were taking medications, or were undergoing continuous positive airway pressure treatment, which minimized the influence of confounding factors on sleep EEG activity.

In conclusion, the characteristics of sleep EEG activity in young and elderly OSAS patients differ; in particular, the slow/fast sigma ratio was associated with OSAS severity only in young patients. The present findings suggest that brain plasticity in young OSAS patients may differ from the plasticity in elderly patients and that this difference may be associated with cognitive decline or increased sleep fragmentation in the elderly group due to OSAS.

\section{REFERENCES}

1. Thomas RJ, Rosen BR, Stern CE, Weiss JW, Kwong KK. Functional imaging of working memory in obstructive sleep-disordered breathing. J Appl Physiol (1985) 2005;98:2226-2234.

2. O'Donoghue FJ, Briellmann RS, Rochford PD, Abbott DF, Pell GS, $\mathrm{Chan} \mathrm{CH}$, et al. Cerebral structural changes in severe obstructive sleep apnea. Am J Respir Crit Care Med 2005;171:1185-1190.

3. Fogel SM, Smith CT. The function of the sleep spindle: a physiological index of intelligence and a mechanism for sleep-dependent memory consolidation. Neurosci Biobehavioral Rev 2011;35:1154-1165.

4. Aeschbach D, Dijk DJ, Borbely AA. Dynamics of EEG spindle frequency activity during extended sleep in humans: relationship to slow-wave activity and time of day. Brain Res 1997;748:131-136.

5. Cote KA, Epps TM, Campbell KB. The role of the spindle in human information processing of high-intensity stimuli during sleep. J Sleep Res 2000;9:19-26.

6. Barakat M, Doyon J, Debas K, Vandewalle G, Morin A, Poirier G, et al. Fast and slow spindle involvement in the consolidation of a new motor sequence. Behav Brain Res 2011;217:117-121.

7. Fogel SM, Smith CT. Learning-dependent changes in sleep spindles and Stage 2 sleep. J Sleep Res 2006;15:250-255.

8. Schönwald SV, Carvalho DZ, de Santa-Helena EL, Lemke N, Gerhardt GJ. Topography-specific spindle frequency changes in obstructive sleep apnea. BMC Neurosci 2012;13:89.

9. Mednick SC, McDevitt EA, Walsh JK, Wamsley E, Paulus M, Kanady JC, et al. The critical role of sleep spindles in hippocampal-dependent memory: a pharmacology study. J Neurosci 2013;33:4494-4504.

10. Mölle M, Bergmann TO, Marshall L, Born J. Fast and slow spindles during the sleep slow oscillation: disparate coalescence and engagement in memory processing. Sleep 2011;34:1411-1421.

11. Crowley K, Trinder J, Kim Y, Carrington M, Colrain IM. The effects of normal aging on sleep spindle and K-complex production. Clin Neurophysiol 2002;113:1615-1622.

12. Himanen S, Virkkala J, Huupponen E, Hasan J. Spindle frequency remains slow in sleep apnea patients throughout the night. Sleep Med 2003; 4:361-366.

13. Carvalho DZ, Gerhardt GJ, Dellagustin G, de Santa-Helena EL, Lemke $\mathrm{N}$, Segal AZ, et al. Loss of sleep spindle frequency deceleration in obstructive sleep apnea. Clin Neurophysiol 2014;125:306-312.

14. Young T, Shahar E, Nieto FJ, Redline S, Newman AB, Gottlieb DJ, et al. Predictors of sleep-disordered breathing in community-dwelling adults: the Sleep Heart Health Study. Arch Intern Med 2002;162:893-900.

15. Trimer R, Cabidu R, Sampaio LL, Stirbulov R, Poiares D, Guizilini S, et al. Heart rate variability and cardiorespiratory coupling in obstructive sleep apnea: elderly compared with young. Sleep Med 2014;15:1324-1341.

16. Ayalon L, Ancoli-Israel S, Drummmond SP. Altered brain activation during response inhibition in obstructive sleep apnea. J Sleep Res 2009; 8:204-208.

17. Dijk DJ, Hayes B, Czeisler CA. Dynamics of electroencephalographic sleep spindles and slow wave activity in men: effect of sleep deprivation. Brain Res 1993;626:190-199.

18. Iber C, Ancoli-Israel S, Chesson AL, Quan SF. The AASM Manual for the Scoring of Sleep and Associated Events: Rules, Terminology and Technical Specifications. Westchester, IL: American Academy of Sleep Medicine; 2007.

19. D’Rozario AL, Dunga GC, Banks S, Lie PY, Wong KKH, Killick R, et al. An automated algorithm to identify and reject artifact for quantitative EEG analysis during sleep in patient with sleep-disordered breathing. Sleep Breath 2015;19:607-615.

20. Edwards BA, O’Driscoll DM, Ali A, Jordan AS, Trinder J, Malhotra A. Aging and sleep: physiology and pathophysiology. Semin Respir Crit Care Med 2010;31:618-633.

21. Redline S, Kirchner HL, Quan SF, Gottlieb DJ, Kapur V, Newman A. The effects of age, sex, ethnicity, and sleep-disordered breathing on sleep architecture. Arch Intern Med 2004;164:406-418.

22. Krieger J, Sforza E, Boudewijns A, Zamagni M, Petiau C. Respiratory effort during obstructive sleep apnea: role of age and sleep state. Chest 1997;112:875-884.

23. Broughton R, Hasan J. Quantitative topographic electroencephalographic mapping during drowsiness and sleep onset. J Clin Neurophysiol 1995;12:372-386.

24. Tamaki M, Matsuoka T, Nittono H, Hori T. Fast sleep spindle (13-15 $\mathrm{Hz}$ ) activity correlates with sleep-dependent improvement in visuomotor performance. Sleep 2008;31:204-211.

25. Ayalon L, Ancoli-Israel S, Drummond SP. Obstructive sleep apnea and age: a double insult to brain function? Am J Respir Crit Care Med 2010; 182:413-419. 